Toxicology and Industrial Health

\title{
Support to Triage and Public Risk Perception Considering Long-Term Response to a Cs-137 Radiological Dispersive Device (RDD) Scenario
}

\begin{tabular}{|r|l|}
\hline Journal: & Toxicology and Industrial Health \\
\hline Manuscript ID & TIH-17-0224.R2 \\
\hline Manuscript Type: & Article \\
\hline Keywords: & Public Health, Consequence, Radiation Risk, Triage, Environment \\
\hline & $\begin{array}{l}\text { A Radiological Dispersive Device (RDD) spreads radioactive material, } \\
\text { complicates the treatment of physical injuries, raises cancer risk and } \\
\text { induces disproportionate fear. Simulating such an event enables more } \\
\text { effective and efficient utilization of the triage and treatment resources of } \\
\text { staff, facilities and space. Fast simulation can give detail on events in } \\
\text { progress, or future events. The resources for triage and treatment of } \\
\text { contaminated trauma victims can differ to those for pure exposure } \\
\text { individuals, whilst discouraging the 'worried well' from presenting in the } \\
\text { crisis phase by media announcement would relieve pressure on hospital } \\
\text { facilities. The proposed methodology integrates capabilities from different } \\
\text { platforms in a convergent way composed of three phases: (a) scenario } \\
\text { simulation; (b) data generation and (c) risk assessment for triage focused } \\
\text { on follow up epidemiological assessment. Simulations typically indicate that } \\
\text { most of the affected population does not require immediate medical } \\
\text { assistance. Medical triage for the few severely injured and the radiological } \\
\text { triage to diminish the contamination with radioactivity will always be the } \\
\text { priority. For this study, however, higher priorities should be given to } \\
\text { individuals from radiological 'warm' and 'hot' zones as required by risk } \\
\text { criteria. The proposed methodology could thus help to: (a) filter and } \\
\text { reduce the number of individuals to be attended; (b) optimize the } \\
\text { prioritization of medical care; (c) reduce or prepare for future costs (d) } \\
\text { effectively locate the operational triage site to avoid possible contamination } \\
\text { on the main facility and (e) provide the scientific data needed to develop } \\
\text { an adequate approach to risk and its proper communication. }\end{array}$ \\
\hline
\end{tabular}




\section{Introduction}

Ionizing radiation is widely used and brings great benefits to various industrial and health care services ranging from radiography and diagnostics to sterilization and brachytherapy. However, the widespread use of radioactive materials leads to occasions of accidental dissemination and radiation exposure, and the potential for theft and misuse. Although the number of immediate mortalities is usually very small in such events, the number of potentially exposed individuals to be screened is relatively high. Consequently, optimizing radiological emergency responses might require special technical procedures to ensure safety. Special emphasis should be placed on the radiological triage of potentially exposed individuals. This has been found to be an important, cumbersome and delicate activity that must be meticulously performed by the occupational health team, which normally heads the process of triage, playing critical roles such as screening and first aids to victims. It is thus expected from occupational health teams to anticipate possible hazards, facilitating safety decisions (Reissman and Howard, 2008). Thus, health team should be familiarized with the effects of ionizing radiation on humans and have some understanding of crisis management tools to efficiently coordinate actions to safely control the situation.

Radioactive material could be dispersed by fire or explosions in places where strong radiation sources exist such as where food, medical instruments or blood are sterilized. However, criminals and terrorists may intentionally cause human harm and disruption with a Radiological Dispersive Device (RDD) by spreading radioactive material in the environment. Typically, an RDD is an explosive device coupled with radioactive material. The explosion adds an immediate threat to human life and property beyond the probable main purpose of the RDD (Saint Yves et al., 2012). The 
combination of blast injuries and radiological contamination can be problematic if procedures to address blast and radiation have only been considered separately. In most plausible scenarios, exposure to radioactive material will not result in the large doses associated to radiation sickness. The primary health concern regarding the public should then be the increased risk of cancer to exposed individuals. Furthermore, hazards from fire, radioactive smoke, shrapnel from explosion, and other chemical or biological agents could also be initially included in the list of major sources of risk. In addition, they are also expected to impact the triage phase of response.

The HotSpot Health Physics code was used to simulate an intentional radioactive dispersion caused by detonation of a radiological (non-nuclear) dispersive device (RDD). A typical RDD uses conventional explosives to spread radioactive material, creating contamination and terrorism (Ring, 2004; Saint Yves et al., 2012). For solid cancers, high doses are necessary to increase the risk significantly above the spontaneous risk, however, for leukemia high relative risks may occur even at low doses (IAEA, 1996). For this reason, the epidemiologic model for leukemia recommended by the Biological Effects of Ionizing Radiation V committee (BEIR V) (Hendee, 1992) was used for risk limits calculations.

Medical attention to injured individuals exposed to radioactive materials can be complex. However, in a typical RDD scenario, the number of individuals exposed to high levels of radiation is not expected to be high (Akashi et al., 2001; Mettler, 2012).

The focus of this study is not exclusively the immediate response to an RDD scenario. It extends to how the early response enables people to be grouped according to their long-term risk of the stochastic effects of cancer, and how this can be used to optimize utilization of resources for urgent medical triage. The triage process of deciding which patients should be treated first is normally based on clinical conditions 
and treatment priorities, where the severely injured will always come first. However, the very resources available to conduct this triage may be unnecessarily confounded by large numbers of people with no immediate symptoms and possibly few long-term symptoms, who may still impact immediate and medium-term capability by attending hospitals and key nodes seeking attention and making contamination control measures necessary. The 'worried well' phenomena have been exhibited in toxic material release hazards such as Goiania and Tokyo (Hildebrand and Bleetman, 2007). One application of the work in this paper is to reduce the concerns of people who are likely to be minimally affected using radio or television broadcasts to announce geographical areas that are of minimal concern, and from where people are best not to seek attention at the current time. There will be apparently healthy people attending triage facilities with enough radiation exposure that not promptly compromise health but increases cancer risk. Those individuals, who with the aid of the forecasts in this work could be diverted away from medical facilities mainly aligned to trauma to other separate facilities designed to quantify and manage the radiological hazard. Minimizing unnecessary cross contamination to victims with open wounds is important. Radiological facilities with the capacity to prevent cross contamination, conduct decontamination, measure internal and external contamination and provide medical countermeasures rarely use similarly trained staff or regions of a hospital as those attending to trauma victims. The appropriate use of available space can be as important as the use of staff. Importantly, this 'pre-triage' would use only knowledge of where public and responders have come from rather than specialist medical knowledge or monitoring equipment. This allows further space, non-clinical staff and the public to share the triage and treatment burden and so hasten throughput. 
Although the long-term risks of cancer may not greatly influence who gets treated first at hospital, the highly emotive perceived risk of cancer in the general population could have a great effect on the numbers to be triaged. This work informs how the triage task could be subdivided between trauma and health physics and how supporting staff can be most effectively used. Although trauma is a priority, there are often separate resources at hospitals that may not be fully utilized treating contaminated trauma casualties and so there would be no reason not to provide early medical treatment to those with greatest long-term risk of developing cancer and it would be socially and politically valuable to do so.

Because the RDD scenario is likely to produce environmental contamination, the occupational team should ideally consider that paths taken by individuals could be contaminated. Since the distribution of radioactive material across the site is not homogeneous, different levels of exposure must be expected at different locations. The plans and system for medical and health response to radiation accidents may vary globally, but the first line is usually emergency medical personnel.

An RDD incident can potentially paralyze a city or state by inflicting significant economic, political and social impact (Rosoff and von Winterfeldt, 2007). Simulating such an event prior to response may provide a valuable picture of the situation, facilitating core activities in response, such as risk communication to the public, strategy and costs evaluation (Rogers et al., 2013).

The purpose of this study is to propose a methodology based on computer modeling to help plan triage procedures in the prompt response to large radiological emergencies aiming: a) reducing the number of individuals to be treated at first sight by using HotSpot code to estimate the number of individuals inside the plume and group them under exposure levels criteria; b) use equations from radioepidemiology to foresee 
cancer development among affected individuals in regard to potential economic, social and political impact.

\section{Methods}

The methodology aims to integrate capabilities from different platforms, a procedure known as convergence, to be performed in three phases: (a) scenario simulation; (b) data generation and (c) risk assessment.

\section{Scenario Simulation}

The Health Physics HotSpot code version 3.0 was developed by the Lawrence Livermore National Laboratory - LLNL, being designed to provide a quick and portable tool to evaluate incidents involving radioactive material (Homann, 2013). HotSpot uses a conservative model to estimate the radiation dose and the concentration of radionuclides as a result of a release into the atmosphere (Homann, 2013; Saint Yves et al., 2012). The model requires less intensive computing and less time than more realistic platforms that may consider terrain and rain, and provides a first quantitative radiological description of the event useful for decision making purposes and planning.

In this study, a simulation was performed to investigate the dispersion of radioactive material in a densely populated area. An RDD event was assumed to occur within an area which hosts major events. The main selections for HotSpot where: (a) general burst mode; (b) radionuclide: Cs-137; (c) activity of $3.70 \times 10^{14} \mathrm{~Bq}$ source; (d) amount of explosive of $25.4 \mathrm{~kg}(10 \mathrm{lb})$ of TNT; (e) atmospheric stability class "E" and (f) Wind speed and direction of $3.0 \mathrm{~m} / \mathrm{s}$ and $200^{\circ}$, respectively. 


\section{Data Generation}

The BEIR V equations for calculating a specific cancer risk were extracted from the Life Span Study (LSS) which is the follow-up study data obtained from a group of survivors of the atomic bombings events at the end of $2^{\text {nd }}$ World War. Measured in cases/100,000 individuals for the purposes of this study, the excess relative risk (ERR) indicates the percentage of morbidity above the baseline record for the population exposed to radiation. It depends on the total equivalent dose (TED) an individual receives, their age at exposure and sex. The radiation doses calculated with HotSpot were inserted into the BEIR V equations for leukemia accounting for age group and sex. The equations are specific for leukemia, regardless of the type (Preston et al., 2004), 2004). The HotSpot calculations provided the location and area of plumes, and when combined with demographic data allow estimates to be made on the number of potentially compromised individuals for an arbitrary site with a typical urban demographic distribution. As a result, the response team could estimate the size of the event, as well as the necessary support and the selection criteria to be adopted in the triage process (prioritization). Additionally, risk assessment and communication to the public would be possible in a way that would positively impact their perception of risk, given the alternative is to rely on divergent and confusing information from the Media and from unofficial sources.

Table 1 presents the equations for determining ERR for leukemia, considering: (1) ERR is a function of: total effective dose ( $D$, in sieverts), latency ( $t$, in years), age at the time of exposure (e, in years) and sex (s); (2) $\mathrm{F}(\mathrm{D})$ is the linear-quadratic function of equivalent dose, and (3) $\beta(\mathrm{t}, \mathrm{s}, e)$ is the Excess relative risk (ERR) parameter (IAEA, 1996). 


\section{TABLE 1 NEAR HERE}

\section{Risk Assessment and Triage for Data Collection}

A modern approach to the triage procedures of individuals in a radiological scenario should consider data from radiological risk assessment and consequence modeling. In such a scenario of environmental contamination the accumulated dose of radiation often has a strong spatial dependence. Thus, accurate locations of individuals as a function of time is important data for the medical purpose of determining doses accumulated along their paths of travel.

HotSpot allows the contamination plume to be projected on the ground so that the affected areas can be readily identified. Those contaminated areas or sites are classified according to their position relatively to the plume (inner, middle and outer) and to the level of radiation exposure: (a) inner - high; (b) middle - moderate and (c) outer - low. Estimating the number of individuals coming from affected areas can be performed by multiplying local population density $(\sigma)$ by the affected areas (A). Having this estimate of the number of affected individuals in each area leads to an appropriate distribution of resources, which speeds up the triage process.

The ERR calculations are estimated according to dose, sex and age, and can be used to define various levels of risk which can be combined with position data. Identifying and projecting the plume area by simulation and applying the ERR calculations can be used to help prevent individuals from moving into dangerous areas, and could even be useful to help identify paths of access or evacuation routes that minimize the overall dose-rate time product. 


\section{Results}

Assuming that the local population density is 5,266 inhabitants $/ \mathrm{km}^{2}$, a total amount of 9,476 individuals was predicted to be within the outer plume area. The corresponding data for each contaminating plume zone was calculated by defining dose limits of $100 \mathrm{mSv}, 50 \mathrm{mSv}$ and $1 \mathrm{mSv}$ equivalent dose, respectively for areas contaminated by the inner, middle and outer plumes respectively. These values are the limits recommended by the International Atomic Energy Agency for responders (100 $\mathrm{mSv}, 50 \mathrm{mSv})$ and public $(1 \mathrm{mSv})$ (IAEA, 2006). Table 2 and 3 show the number of individuals in three age groups over the three plume zones and ERR by gender, age (e) and location (isodoses line) respectively.

\section{TABLE 2 NEAR HERE}

\section{TABLE 3 NEAR HERE}

\section{Discussion}

The simulated scenario was built based on data provided by first responders and then input to HotSpot. The results listed in Table 2 show that most of the population is located in the outer plume region, representing nearly $93.9 \%$ of the affected population, 9,478 out of 10,100 individuals, who may not require any immediate medical assistance.

Higher priority should be given to assist more seriously exposed individuals located in the inner and middle plumes as required by risk criteria (Reissman and 
Howard, 2008). According to data from Table 2, the total number of individuals that are expected to require medical attention would be from areas affected by the middle and inner plumes, and would account for $6.1 \%$ of the affected population of 10,100 individuals. Such individuals from these two innermost parts of the plume should be initially treated according to priorities related to sex and age, and then evaluated and monitored in epidemiological programs. In case of severe traumas evaluated by the medical staff, priority should be given without considering potential contamination (Division, 2010), although an assessment by radiological monitoring is recommended before leaving the triage area. Otherwise, higher priorities for immediate medical care will be given to individuals located in the inner and middle plumes, respectively (Table 2). For each plume area, any leukemia based prioritization should be given according to table 3 , being higher for men and younger individuals. This prioritization would significantly reduce the load on the triage area. However, for achieving this goal, the triage forms should include information on the entire path of everyone to the triage site.

\section{Conclusion}

The correct designation of potentially exposed individuals in a radiological emergency scenario has a positive impact on the allocation of resources and quality of medical care. The proposed combination of HotSpot and BEIR V equations according to convergence methodology could efficiently help improve the triage process by promptly providing valuable information that would aid in efforts to: (a) filter and reduce the number of individuals to be attended; (b) optimize the prioritization of medical care; (c) reduce operational costs and (d) provide the scientific data needed to develop an adequate approach to risk and its proper communication to the public. 


\section{References}

Akashi M, Hirama T, Tanosaki S, et al. (2001) Initial symptoms of acute radiation syndrome in the JCO criticality accident in Tokai-mura. Journal of Radiation Research 42 Suppl: S157-166.

Division DEP. (2010) The ambulance service guidance on dealing with radiological incidents and emergencies. In: Guidance NEP (ed). http://webarchive.nationalarchives.gov.uk/20130107105354/http:/www.dh.gov.u k/prod_consum_dh/groups/dh_digitalassets/@dh/@en/@ps/documents/digitalas set/dh_114466.pdf: NHS.

Hendee WR. (1992) Estimation of radiation risks. BEIR V and its significance for medicine. Journal of the American Medical Association 268(5): 620-624.

Hildebrand S and Bleetman A. (2007) Comparative study illustrating difficulties educating the public to respond to chemical terrorism. Prehospital Disaster Medicine 22(1): 35-41.

Homann SG. (2013) HotSpot Health Physics Codes Version 3.0 User's Guide. CA, USA.: Lawrence Livermore National Laboratory.

IAEA. (1996) IAEA-TECDOC-870 - Methods for Estimating the Probability of Cancer from Occupational Radiation Exposure. In: Section RS (ed). Vienna: IAEA.

IAEA. (2006) Manual for First Responders to a Radiological Emergency. Incident and Emergency Centre. Vienna: International Atomic Energy Agency.

Mettler FA. (2012) Medical effects and risks of exposure to ionising radiation. Journal of Radiological Protection 32(1): N9-N13.

Preston DL, Pierce DA, Shimizu Y, et al. (2004) Effect of recent changes in atomic bomb survivor dosimetry on cancer mortality risk estimates. Radiation Reseach 162(4): 377-389.

Reissman DB and Howard J. (2008) Responder safety and health: preparing for future disasters. Mount Sinai Journal of Medicine 75(2): 135-141.

Ring JP. (2004) Radiation risks and dirty bombs. Health Physics 86(2 Suppl): S42-47.

Rogers MB, Amlot R and Rubin GJ. (2013) The impact of communication materials on public responses to a radiological dispersal device (RDD) attack. Biosecurity and Bioterrorism 11(1): 49-58.

Rosoff $\mathrm{H}$ and von Winterfeldt D. (2007) A risk and economic analysis of dirty bomb attacks on the ports of Los Angeles and Long Beach. Risk Analysis 27(3): 533546.

Saint Yves TLA, Cabral PAM, Brum T, et al. (2012) Terrorist Radiological Dispersive Device (RDD) Scenario and Cancer Risk Assessment. Human and Ecological Risk Assessment 18(5): 971-983. 
Table 1. Functions based on dose, sex, time and age for calculating the ERR for leukemia. The Equations for male and female are from BEIR V committee (IAEA, 1996).

\begin{aligned} & MALE \\ & $\operatorname{ERR}(\mathrm{D}, \mathrm{t}, e, \mathrm{~s})=\mathrm{F}(\mathrm{D}) \beta(\mathrm{t}, e, \mathrm{~s})=0.33\left(\mathrm{D}+0.79 \mathrm{D}^{2}\right) \exp [-0.17(\mathrm{t}-25)] e<20 \\ & \operatorname{ERR}(\mathrm{D}, \mathrm{t}, e, \mathrm{~s})=\mathrm{F}(\mathrm{D}) \beta(\mathrm{t}, e, \mathrm{~s})=0.48\left(\mathrm{D}+0.79 \mathrm{D}^{2}\right) \exp [-0.13(\mathrm{t}-25)] 20 \leq e<40 \\ & \operatorname{ERR}(\mathrm{D}, \mathrm{t}, e, \mathrm{~s})=\mathrm{F}(\mathrm{D}) \beta(\mathrm{t}, e, \mathrm{~s})=1.31\left(\mathrm{D}+0.79 \mathrm{D}^{2}\right) \exp [-0.07(\mathrm{t}-25)] e \geq 40 \\ &$ FEMALE \\ & $\operatorname{ERR}(\mathrm{D}, \mathrm{t}, e, \mathrm{~s})=\mathrm{F}(\mathrm{D}) \beta(\mathrm{t}, e, \mathrm{~s})=0.66\left(\mathrm{D}+0.79 \mathrm{D}^{2}\right) \exp [-0.07(\mathrm{t}-25)] e<20 \\ & \operatorname{ERR}(\mathrm{D}, \mathrm{t}, e, \mathrm{~s})=\mathrm{F}(\mathrm{D}) \beta(\mathrm{t}, e, \mathrm{~s})=0.97\left(\mathrm{D}+0.79 \mathrm{D}^{2}\right) \exp [-0.03(\mathrm{t}-25)] 20 \leq e<40 \\ & \operatorname{ERR}(\mathrm{D}, \mathrm{t}, e, \mathrm{~s})=\mathrm{F}(\mathrm{D}) \beta(\mathrm{t}, e, \mathrm{~s})=2.64\left(\mathrm{D}+0.79 \mathrm{D}^{2}\right) \exp [0.03(\mathrm{t}-25)] e \geq 40 \\ &$\hline\end{aligned}


Table 2. Affected areas and the approximate number of individuals within the contamination plumes taken by the mathematical relation between the area (A) and the local population density $(\sigma)$.

\begin{tabular}{lcccc}
\hline \multicolumn{1}{c}{ Parameters } & \multicolumn{3}{c}{ Plumes } & \multirow{2}{*}{ Total (approx.) } \\
\cline { 2 - 4 } & inner & middle & outer & \\
\hline dose $(\mathbf{S v})$ & 0.100 & 0.050 & 0.001 & - \\
plume area $\left(\mathbf{k m}^{\mathbf{2}}\right)$ & 0.032 & 0.086 & 1.8 & 10,100 \\
individuals & 169 & 452 & 9,478 & 2,687 \\
individuals $(\boldsymbol{e}<\mathbf{2 0})$ & 45 & 120 & 2,521 & 3,282 \\
individuals $(\mathbf{2 0} \leq \boldsymbol{e}<\mathbf{4 0})$ & 55 & 147 & 3,080 & 4,131 \\
individuals $(\boldsymbol{e} \geq \mathbf{4 0})$ & 69 & 185 & 3,877 & \\
\hline
\end{tabular}


Table 3. ERR calculated based on equations 1 to 6 by gender, age (e) and location (isodoses line).

\begin{tabular}{ccccc}
\hline \multirow{2}{*}{ Parameters } & \multicolumn{3}{c}{ Plumes } \\
\cline { 2 - 5 } & & inner & middle & outer \\
\hline sex & age (years) & & ERR & \\
\hline male & $20<e<40$ & 0.965 & 0.786 & 0.015 \\
& $e \geq 40$ & 0.683 & 0.465 & 0.009 \\
female & $20 \leq e<40$ & 0.206 & 0.329 & 0.006 \\
& $e \geq 40$ & 0.145 & 0.166 & 0.003 \\
& & & 0.099 & 0.002 \\
& & & & 0.074 \\
\end{tabular}

